\title{
Prediction of Human Papillomavirus Status and Overall Survival in Patients with Untreated Oropharyngeal Squamous Cell Carcinoma: Development and Validation of CT-Based Radiomics
}

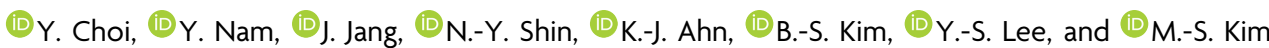

\begin{abstract}
BACKGROUND AND PURPOSE: Human papillomavirus is a prognostic marker for oropharyngeal squamous cell carcinoma. We aimed to determine the value of CT-based radiomics for predicting the human papillomavirus status and overall survival in patients with oropharyngeal squamous cell carcinoma.
\end{abstract}

MATERIALS AND METHODS: Eighty-six patients with oropharyngeal squamous cell carcinoma were retrospectively collected and grouped into training $(n=61)$ and test $(n=25)$ sets. For human papillomavirus status and overall survival prediction, radiomics features were selected via a random forest-based algorithm and Cox regression analysis, respectively. Relevant features were used to build multivariate Cox regression models and calculate the radiomics score. Human papillomavirus status and overall survival prediction were assessed via the area under the curve and concordance index, respectively. The models were validated in the test and The Cancer Imaging Archive cohorts $(n=78)$.

RESULTS: For prediction of human papillomavirus status, radiomics features yielded areas under the curve of $0.865,0.747$, and 0.834 in the training, test, and validation sets, respectively. In the univariate Cox regression, the human papillomavirus status (positive: hazard ratio, $0.257 ; 95 \% \mathrm{Cl}, 0.09-0.7 ; P=.008$ ), T-stage ( $\geq \mathrm{III}$ : hazard ratio, 3.66; 95\% Cl, 1.34-9.99; $P=.011$ ), and radiomics score (high-risk: hazard ratio, 3.72; 95\% Cl, 1.21-11.46; $P=.022$ ) were associated with overall survival. The addition of the radiomics score to the clinical Cox model increased the concordance index from 0.702 to $0.733(P=.01)$. Validation yielded concordance indices of 0.866 and 0.720 .

CONCLUSIONS: CT-based radiomics may be useful in predicting human papillomavirus status and overall survival in patients with oropharyngeal squamous cell carcinoma.

ABBREVIATIONS: AUC = area under the curve; HPV = human papillomavirus; OPSCC = oropharyngeal squamous cell carcinoma; OS = overall survival; rad-scores $=$ radiomics scores; $\mathrm{TCIA}=$ The Cancer Imaging Archive

O ropharyngeal squamous cell carcinoma (OPSCC) is one of the most rapidly growing subtypes of head and neck cancers, primarily due to the increased incidence of human papillomavirus (HPV)-related OPSCC. ${ }^{1} \mathrm{HPV}$ is a well-established

Received January 20, 2020; accepted after revision July 3.

From the Departments of Radiology (Y.C., Y.N., J.J., N.-Y.S, K.-J.A., B.-S.K.), Hospital Pathology (Y.-S.L.), and Otolaryngology-Head and Neck Surgery (M.-S.K.), Seoul St. Mary's Hospital, College of Medicine, The Catholic University of Korea, Seoul,

Republic of Korea; and Division of Biomedical Engineering (Y.N.), Hankuk University of Foreign Studies, Yongin-Si, Gyeonggi-do, Republic of Korea.

Please address correspondence to Kook-Jin Ahn, MD, PhD, Department of Radiology, Seoul St. Mary's Hospital, College of Medicine, The Catholic University of Korea, 222 Banpo-daero, Seocho-gu, Seoul (06591), Korea; e-mail:

ahn-kj@catholic.ac.kr

Indicates article with supplemental on-line table.

Indicates article with supplemental on-line photo.

http://dx.doi.org/10.3174/ajnr.A6756 prognostic factor for OPSCC, with a positive HPV status carrying a better prognosis. ${ }^{2-4}$ This distinction in HPV status has contributed to the recent change in OPSCC staging in the 8th edition of the American Joint Committee on Cancer Staging Manual..$^{5-7}$ Because HPV status has substantial clinical implications, tissue biopsy from a primary or neck site and a test for p16/HPV are indicated. Although biopsy is generally straightforward in the head and neck, it is conceivable that image-based differentiation of OPSCC based on HPV status could become relevant, especially if it comes with cost savings.

Imaging of head and neck cancer is often difficult due to anatomic complexity, overlapping tissue densities, and artifacts (ie, motion, dental amalgam, and so forth). Thus, describing imaging features can be challenging. Recently, the advent of radiomics in image-based research has allowed the quantification of such complex imaging features into standardized high-throughput data, 
the subsequent analysis of which could aid clinical decisionmaking. ${ }^{8}$

Before radiomics, diffusion-weighted MR imaging indicated that HPV-positive OPSCC had lower apparent diffusion coefficients than its counterpart, ${ }^{9}$ a finding that was presumed to be due to low stromal volume ${ }^{10}$ and abundant lymphoid cells ${ }^{11}$ associated with HPV-positive OPSCC. Previous CT-based radiomics studies reported promise in prognostication, ${ }^{12}$ prediction of HPV status, ${ }^{9}$ and both prediction and prognostication in head and neck cancers. ${ }^{13,14}$

We hypothesized that CT-based radiomics could fulfill both purposes-HPV status and overall survival (OS) prediction. Therefore, the aim of this study was to determine whether pretreatment CT-based radiomics of primary OPSCC could predict the HPV status and OS of patients initially diagnosed with OPSCC.

\section{MATERIALS AND METHODS \\ Patients}

This single-center retrospective cohort study was approved by the institutional review board of Seoul St. Mary's Hospital, and the need to obtain informed consent was waived. Between January 2009 and September 2019, one hundred twenty-five patients initially diagnosed with OPSCC were retrospectively reviewed. The inclusion criteria were the following: 1) pathologically confirmed OPSCC, 2) known HPV status, and 3) available pretreatment contrast-enhanced neck CT images. Thirty-nine patients were excluded for the following reasons: 1) primary tumor not visible on CT $(n=8), 2)$ beam-hardening artifacts hampering appropriate image analysis $(n=26)$, and 3$)$ other underlying malignancy $(n=3)$ or distant metastases $(n=2)$ at the time of OPSCC diagnosis. Finally, a total of 86 eligible patients were selected for analysis. Clinical information, including age at diagnosis, sex, smoking history, cancer staging (American Joint Committee on Cancer Staging Manual, 8th edition), and HPV status were retrieved via the electronic medical records of our institution. OS was defined as the interval from the date of initial diagnosis to the date of death or last documented clinical visit. The cohort was grouped into training and test cohorts (7:3 ratio) by random stratified sampling so that the survival status (dead or alive) in each group was evenly distributed. The review of patients' medical records was finalized on October 2, 2019.

\section{Independent External Validation Dataset}

An independent external cohort $(n=137)$ was retrieved from The Cancer Imaging Archive (TCIA), an open repository of various oncologic images. ${ }^{15}$ We specifically selected the head and neck cancer dataset previously published by Aerts et al, ${ }^{16}$ who publicly shared CT-segmented primary OPSCC for reproducibility purposes. The patients were subjected to the same selection process as described above. After screening for eligibility criteria, 59 patients were excluded for the following reasons: 1) no OPSCC $(n=49), 2)$ unknown HPV status $(n=8)$, and 3$)$ errors in importing segmentation $(n=2)$. Finally, 78 eligible patients were included as an external validation dataset.

\section{HPV Status Assessment}

The formalin-fixed and paraffin-embedded tissues were prepared in 4-mm-thick sections, which were then mounted onto 3-aminopropylmethoxysilane-coated slides. In situ hybridization was processed on an automated BenchMark system (Ventana Medical Systems) via INFORMH HPV III Family 16 Probe (cocktail of HPV subtypes 16, 18, 31, 33, 35, 39, 45, 51, 52, 56, and 66; Ventana Medical Systems) as recommended by the manufacturer. After removal of the paraffin wax, the tissue underwent protease digestion and hybridization with a probe. The probe-target complex was detected by the action of alkaline phosphatase on the 5-bromo-4-chloro-3-indolyl phosphate, which yielded dark blue with a pink counterstain for the HPVnegative cells due to nuclear fast red. The nuclear hybridization signal was evaluated by a head and neck pathologist (Y.-S.L.).

\section{CT Image Acquisition}

CT imaging of the neck was acquired using 2 different 128-channel multidetector CT scanners (Somatom Definition AS+ and Somatom Definition; Siemens). The CT protocol included precontrast and contrast-enhanced images after intravenous injection of $100 \mathrm{~mL}$ of iodinated contrast agent (60-second delay) (370 mg of iodine/mL, Ultravist, iopromide; Bayer HealthCare) at $2.5 \mathrm{~mL} / \mathrm{s}$ via an automatic flow power injector $\left(\mathrm{MEDRAD}^{\circledR}\right.$ Stellant, Bayer, Leverkusen, Germany). The scan parameters for both scanners were the following: $x$-ray voltage, $120 \mathrm{kV}$ (peak); automatic tube current modulation (CARE Dose, syngo CT; Siemens); rotation time, 1 second; pitch, 0.8 ; detector collimation, $0.6 \mathrm{~mm}$; matrix size, $512 \times 512$ pixels; FOV, $25 \mathrm{~cm}$; and section thickness, $3 \mathrm{~mm}$. The $\mathrm{z}$-axis encompassed the skull base to the aortic arch.

\section{Image Analysis and Radiomics Feature Extraction}

All image analyses were performed using syngo.via Frontier software (Siemens). Primary sites of OPSCC were semiautomatically segmented using the "lesion segmentation" function within the software, ${ }^{17}$ which yielded a 3D ROI contour; minimal additional adjustment of the ROI was needed to avoid beam-hardening artifacts and other soft tissues surrounding the tumor. 3D ROI segmentation included both solid and necrotic portions. The semiautomatic segmentation required approximately 2 seconds of processing time per patient. A radiologist (Y.C.) with 7 years of experience in head and neck imaging segmented all ROIs; another radiologist (K.-J.A.) with 20 years of experience in head and neck imaging reviewed and confirmed the ROIs, and any discrepancy was resolved by consensus. Both radiologists were blinded to the patients' clinical information during image analysis. Representative images of ROI segmentations are provided in Fig 1.

The ROIs were initially resampled to an isometric voxel size of $1 \mathrm{~mm}$. Radiomic features were extracted using PyRadiomicsa publicly maintained platform of radiomic features ${ }^{18}$ that were embedded in syngo.via Frontier. Six different classes of features were automatically extracted, yielding a total of 854 features per patient. The detailed information regarding these features is publicly available (https://pyradiomics.readthedocs.io/en/latest/). 


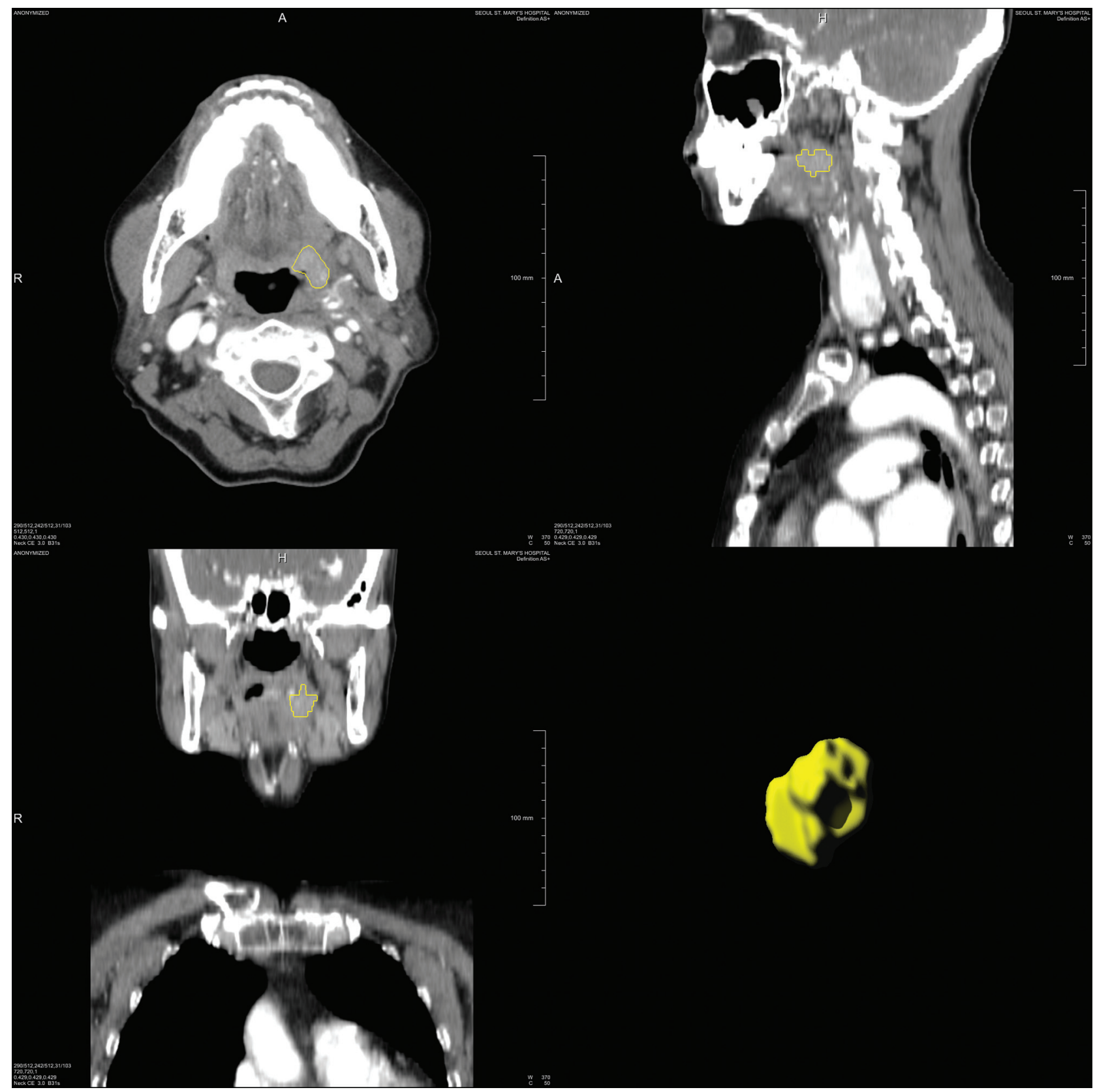

FIG 1. Representative contrast-enhanced CT images with a 3D ROI in a 73-year-old female patient. Volumetric rendering of segmentation is illustrated as a yellow contour (lower right).

\section{Radiomics Feature Selection}

For HPV-status prediction, radiomics features were selected via Boruta, which is a random forest-based wrapper algorithm for all-relevant feature selection. ${ }^{19}$ Random forest generates an importance measure for each feature, allowing minimal parameter adjustment. In estimating feature importance, we applied the Boruta algorithm repetitively, and irrelevant features were consecutively excluded. The Boruta algorithm reached statistical significance by continuously calculating all possible feature combinations, producing an all-relevant subset of features.

Regarding OS prediction, least absolute shrinkage and selection operator-Cox regression were used for dimensionality reduction of radiomics features. ${ }^{20}$ The selected features were weighted by their respective coefficients to calculate radiomics scores (rad-scores) for individual patients, with the median radscore being used to dichotomize patients into high-risk and lowrisk groups. All feature selections were performed in the training cohort to remove the possibility of overfitting.

\section{Statistical Analysis}

For HPV-status prediction, the Boruta-selected radiomic features from the training cohort were fitted into generalized linear models to plot receiver operating characteristic curves with calculation of the area under the curve (AUC). The same features were applied in the test and TCIA cohorts for internal and external validation, respectively, to calculate their respective AUCs. The optimal cutoff 


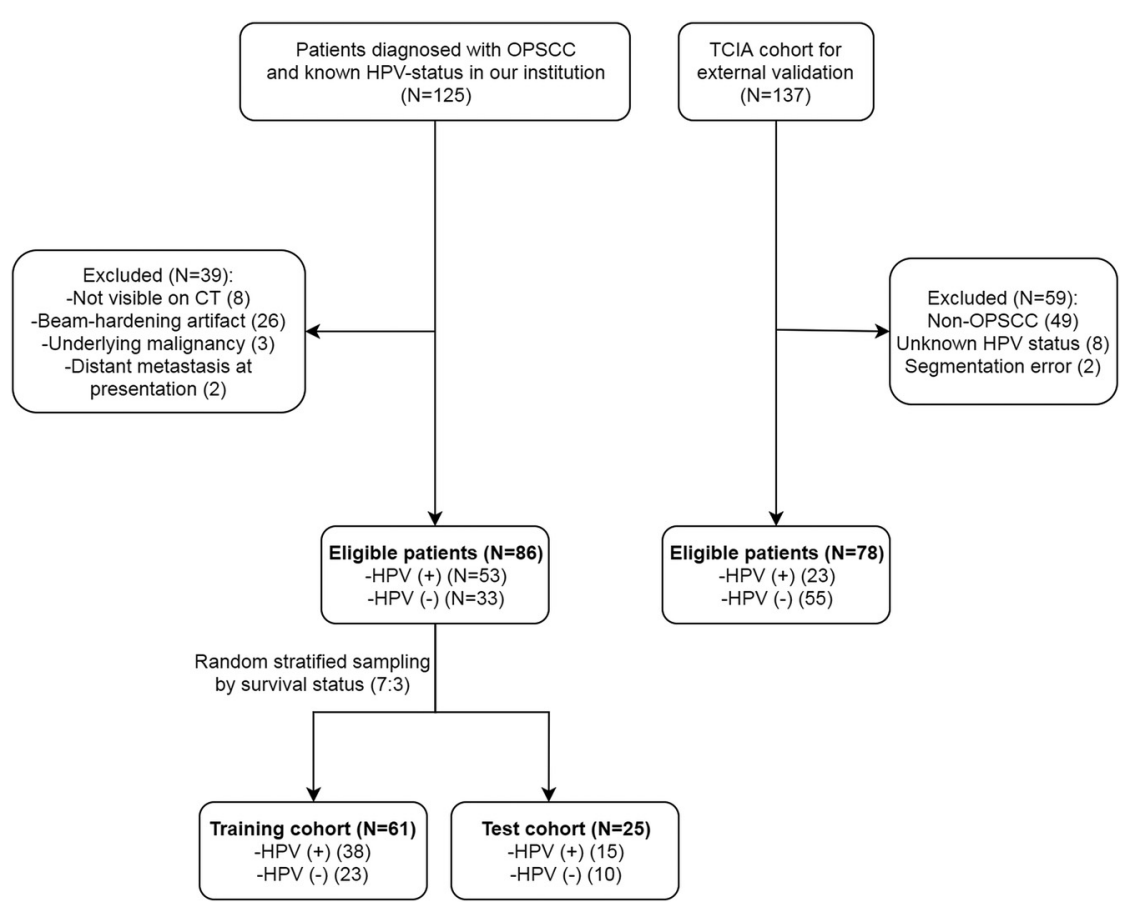

FIG 2. A flow diagram illustrating the patient-selection process. threshold yielding the best AUC was obtained via the Youden index. Receiver operating characteristic curves with AUCs and 95\% CIs were generated with 2000-times stratified bootstrapping. Comparisons among AUCs were performed using the DeLong test.

For OS prediction, a univariate Cox proportional hazard analysis was performed using variables including HPV status, smoking history, age (65 years of age or older), sex, T-stage (I/II versus III/IV), N-stage (0/I versus II/III), and rad-score (high-risk versus low-risk). Kaplan-Meier survival curves were plotted for each of these variables. Logrank tests were used to compare the survival curves. Moreover, a nomogram for 2- and 5-year OS probabilities was constructed using the HPV status, T-stage, N-stage, and rad-score.

Multivariable Cox proportional hazards analyses were also performed to build 3 Cox regression models based on cancer stage (ie, T- and N-
Table 1: Clinical characteristics of the 2 study cohorts

\begin{tabular}{|c|c|c|}
\hline Characteristics & $\begin{array}{c}\text { Institutional } \\
\text { Cohort }(n=86)\end{array}$ & $\operatorname{TCIA}(n=78)$ \\
\hline Age (mean) (yr) & $60.9 \pm 8.6$ & $60.2 \pm 7.6$ \\
\hline \multicolumn{3}{|l|}{ Sex (No.) (\%) } \\
\hline Male & $78(90.7)$ & $61(78.2)$ \\
\hline Female & $8(9.3)$ & $17(21.8)$ \\
\hline \multicolumn{3}{|l|}{ Smoking history (No.) (\%) } \\
\hline Smoker & $50(58.1)$ & NA \\
\hline Nonsmoker & 36 (41.9) & \\
\hline \multicolumn{3}{|l|}{ HPV status (No.) (\%) } \\
\hline Positive & $53(61.6)$ & $23(29.5)$ \\
\hline Negative & $33(38.4)$ & $55(70.5)$ \\
\hline \multicolumn{3}{|l|}{ T-stage (No.) (\%) } \\
\hline I & $19(22.1)$ & $13(16.7)$ \\
\hline II & 41 (47.7) & $24(30.8)$ \\
\hline III & $20(23.3)$ & $10(12.8)$ \\
\hline IV & $6(7.0)$ & 31 (39.7) \\
\hline \multicolumn{3}{|l|}{ N-stage (No.) (\%) } \\
\hline 0 & $18(20.9)$ & $20(25.6)$ \\
\hline I & $32(37.2)$ & $11(14.1)^{\prime}$ \\
\hline II & $33(38.4)$ & $45(57.7)$ \\
\hline III & $3(3.5)$ & $2(2.6)$ \\
\hline \multicolumn{3}{|l|}{ Overall stage (No.) (\%) } \\
\hline I & $25(29.1)$ & $5(6.4)$ \\
\hline ॥ & $25(29.1)$ & $7(9)$ \\
\hline III & $23(26.7)$ & $10(12.8)$ \\
\hline IVA & $11(12.8)$ & $49(62.8)$ \\
\hline IVB & $2(2.3)$ & $7(9)$ \\
\hline No. of deaths (No.) (\%) & $24(27.9)$ & $45(57.7)$ \\
\hline $\begin{array}{l}\text { Overall survival (mean) } \\
\text { (day) }\end{array}$ & $1290.6 \pm 883.4$ & $2243.4 \pm 1370.7$ \\
\hline \multicolumn{3}{|l|}{ Treatment (No.) (\%) } \\
\hline Surgery & $60(69.8)$ & $3(3.8)$ \\
\hline Chemotherapy & $56(65.1)$ & NA \\
\hline Radiation therapy & $60(69.8)$ & $78(100)$ \\
\hline
\end{tabular}

Note:-NA indicates not applicable. stage) alone (Cox model 1), cancer stage combined with the HPV status (Cox model 2), and cancer stage combined with both the HPV status and rad-score (Cox model 3). Harrell concordance indices were calculated for each model with likelihood ratio tests. Cox model 3 was both internally and externally validated in the test and TCIA cohorts to calculate the respective concordance indices. Finally, prediction error curves over survival times were plotted for the institutional and TCIA cohorts with an integrated Brier score, which ranges from 0 (perfect model) to 0.25 (meaningless model). All statistical analyses were performed using $\mathrm{R}$ statistical software (Version 3.5.1; http://www.r-project.org/) with "glmnet," "survival," "pROC," "pec," "caret" (for random stratified sampling), and "rms" (for nomogram) packages. The statistical significance was set at $P<.05$.

\section{RESULTS \\ Patients}

A flow diagram of the patient selection process is provided in Fig 2. The clinical characteristics of the study population are summarized in Table 1 . Among the 86 patients in the institutional cohort (mean age, $60.9 \pm 8.6$ years; male, 90.7\% [78/ 86]), $53(61.6 \%)$ and $33(38.4 \%)$ patients were HPV-positive and -negative, respectively. Stage II was the most prevalent for both T-stage $(47.7 \%, 41 / 86)$ and $\mathrm{N}$-stage $(38.4 \%, 33 / 86)$. The training and test cohorts consisted of 61 and 25 patients, respectively; their clinical characteristics are provided in the On-line Table.

For the TCIA cohort (mean age, $60.2 \pm 7.6$ years; male, $78.2 \%$ [61/78]), 23 (29.5\%) and 55 patients (70.5\%) were HPV-positive and -negative, respectively. T-stage IV $(39.7 \%, 31 / 78)$ and $\mathrm{N}$ stage II $(57.7 \%, 45 / 78)$ were the most prevalent. 
Table 2: Relevant features for the prediction of human papillomavirus status and overall survival

\begin{tabular}{lcc}
\hline & $\begin{array}{c}\text { Mean Variable } \\
\text { Importance }\end{array}$ & $\begin{array}{c}\text { Norm Hits }{ }^{\mathrm{a}} \text { / } \\
\text { Coefficients }\end{array}$ \\
\hline $\begin{array}{l}\text { Selected features by Boruta for prediction (Norm Hits) } \\
\text { original_shape_Flatness }\end{array}$ & 4.753 & 0.812 \\
original_shape_SphericalDisproportion & 5.012 & 0.828 \\
wavelet_HLH_firstorder_Mean & 9.017 & 0.965 \\
wavelet_HLH_firstorder_Uniformity & 3.606 & 0.678 \\
wavelet_HLH_glcm_ClusterShade & 4.692 & 0.804 \\
wavelet_LHH_glcm_Idm & 3.296 & 0.600 \\
wavelet_LHH_glcm_Imcl & 4.201 & 0.753 \\
wavelet_LHL_glszm_SmallAreaHighGrayLevelEmphasis & 11.378 & 0.989 \\
wavelet_LLH_glcm_Imc2 & 4.503 & 0.792 \\
Selected features by LASSO-Cox for OS (coefficient) & & \\
original_shape_SphericalDisproportion & & $3.20 \mathrm{E}-01$ \\
original_firstorder_Minimum & & $-2.36 \mathrm{E}-03$ \\
original_firstorder_1OPercentile & & $-1.75 \mathrm{E}-05$ \\
\hline
\end{tabular}

Note:-LASSO indicates least absolute shrinkage and selection operator; glcm, gray-level co-occurrence matrix; glszm, gray-level size-zone matrix; idm, inverse difference moment; imcl, informational measure of correlation 1; imc2, informational measure of correlation 2.

${ }^{a}$ Fraction of random forest runs (Norm Hits) in which they were more important than the most important shadow value.

\section{Relevant Radiomics Features}

For each patient in the training cohort, 854 features from 6 different classes (first-order statistics, shape-based, graylevel cooccurrence matrix, gray-level run-length matrix, gray-level size-zone matrix, neighboring gray-tone difference matrix, and gray-level dependence matrix) were extracted. Among these, the Boruta algorithm selected 9 relevant features ( 2 shape-based, 2 first-order, 4 gray-level co-occurrence matrices, and 1 gray-level size-zone matrix) for the prediction of HPV status (Table 2).

As for OS prediction, least absolute shrinkage and selection operator-Cox regression yielded 3 radiomic features, including 1 shape-based and 2 firstorder features. From these, the radscore was calculated using the follow-

Table 3: Sensitivity, specificity, and accuracy of radiomics features in predicting human papillomavirus status

\begin{tabular}{lcccc}
\multicolumn{1}{c}{ Cohort } & Sensitivity (95\% CI) & Specificity (95\% CI) & Accuracy (95\% CI) & AUC (95\% CI) \\
\hline Training $(n=61)$ & $76.3 \%(55.3 \%-100 \%)$ & $91.3 \%(56.5 \%-100 \%)$ & $80.3 \%(70.5 \%-90.2 \%)$ & $0.865(0.777-0.953)$ \\
Test $(n=25)$ & $100 \%(40 \%-100 \%)$ & $60.0 \%(30.0 \%-100 \%)$ & $80.0 \%(60.0 \%-92.0 \%)$ & $0.747(0.533-0.961)$ \\
TCIA $(n=78)$ & $82.6 \%(60.9 \%-100 \%)$ & $80.0 \%(52.7 \%-94.6 \%)$ & $80.8 \%(65.4 \%-89.7 \%)$ & $0.834(0.738-0.930)$ \\
\hline
\end{tabular}
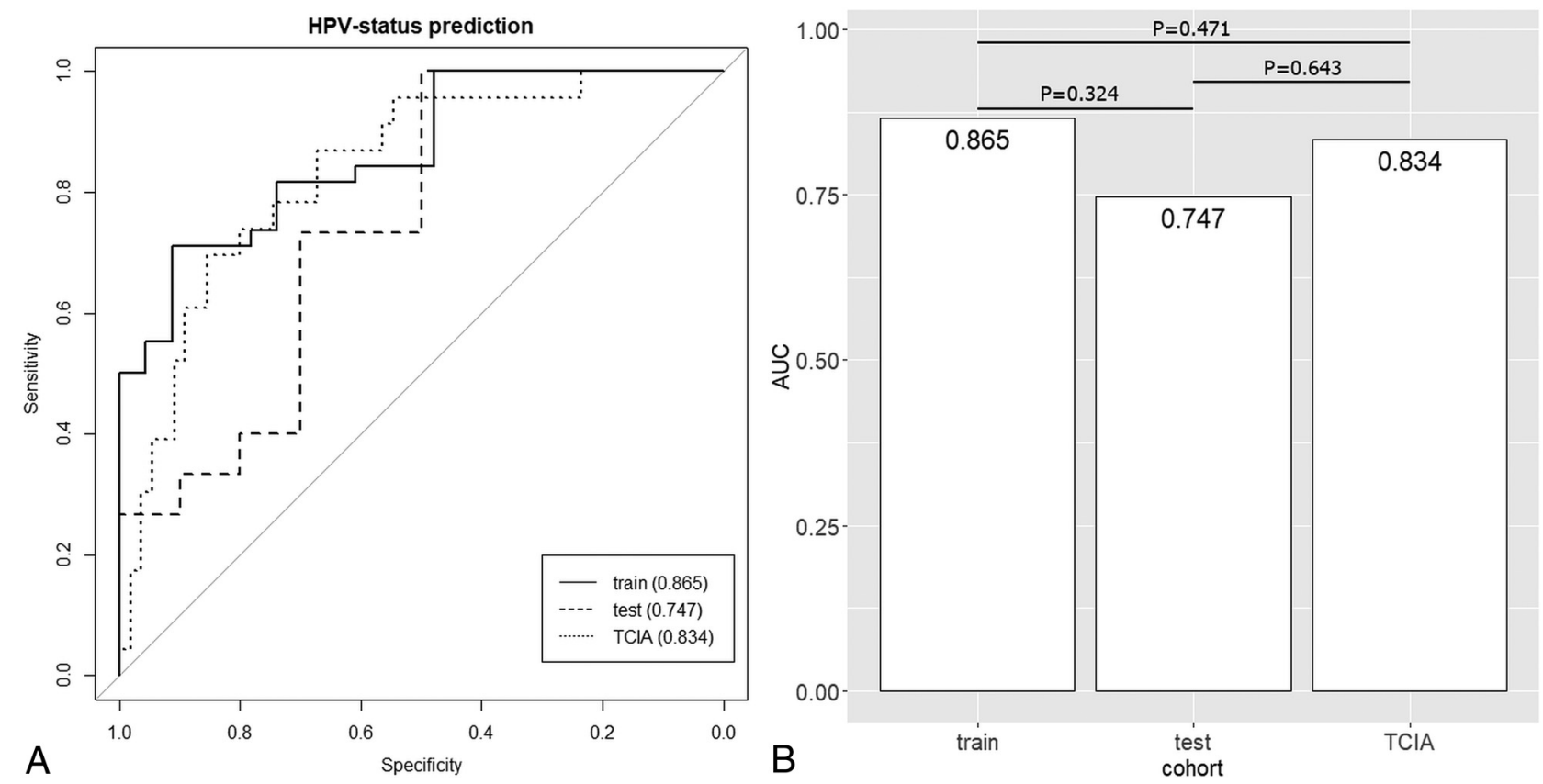

FIG 3. Receiver operating characteristic curves with AUC values for HPV-status prediction in the training, test, and TCIA cohorts (A). The $P$ values calculated from the DeLong test between a pair of AUCs are presented above each line segment (B). 
ing formula with Cox proportional hazard weights given to each feature as previously described: ${ }^{12}$

$0.32 \times$ Original_Shape_SphericalDisproportion $+(-2.363-$ $\left.\times 10^{-3}\right) \times$ Original_Firstorder_Minimum $+\left(-1.753 \times 10^{-5}\right) \times$ Original_Firstorder_10Percentile.

\section{Performances of Radiomics Features in HPV Status Prediction}

The generalized linear models fitted with 9 radiomics features in the training cohort yielded an AUC of 0.865 (95\% CI, 0.777-0.953) with a sensitivity and specificity of $76.3 \%$ and $91.3 \%$, respectively (Table 3). The internal validation, which was performed on a

Table 4: Cox proportional hazards analyses and model validation

\begin{tabular}{llcc}
\hline & HR & 95\%Cl/C-Index & $P$ Value \\
\hline Clinical variable (95\% CI) & & & \\
HPV status, positive & 0.26 & $(0.09-0.7)$ & $.008^{\mathrm{a}}$ \\
Smoking history, smoker & 1.9 & $(0.66-5.44)$ & .23 \\
Age, older than 65 years & 0.97 & $(0.34-2.78)$ & .96 \\
Sex, female & 0.51 & $(0.07-3.83)$ & .51 \\
T-stage, $\geq$ III & 3.66 & $(1.34-9.99)$ & $.01^{\mathrm{a}}$ \\
N-stage, $\geq \mathrm{II}$ & 1.62 & $(0.62-4.19)$ & .32 \\
Overall stage, $>$ IV & 2.7 & $(0.95-7.68)$ & .06 \\
Rad-score, high-risk & 3.72 & $(1.21-11.46)$ & $.02^{\mathrm{a}}$ \\
Multivariate model (C-index) & & & \\
Cancer stage (model 1) & & 0.621 & .03 \\
Cancer stage + HPV status (model 2) & & 0.702 & .01 \\
Cancer stage + HPV status + rad-score (model 3) & & 0.733 & .01 \\
Validation (C-index) & & & \\
Internal validation on test set & & 0.866 & .01 \\
External validation on TCIA set & & 0.72 & $<.001$ \\
\hline
\end{tabular}

Note:-HR indicates hazard ratio; $\mathrm{C}$ index, concordance index.

astatistically significant. separate test set (30\% of the initial cohort), yielded an AUC of 0.747 (95\% CI, 0.533-0.961) with a sensitivity and specificity of $100 \%$ and $60 \%$, respectively. The external validation yielded an AUC of 0.834 (95\% CI, 0.738-0.930) with a sensitivity and specificity of $82.6 \%$ and $80 \%$, respectively. Comparison of the AUCs revealed no significant differences (training versus test, $P=.324$; training versus TCIA, $P=.643$; test versus TCIA, $P=.471$ ) (Fig 3 ).

\section{Performance of Radiomics Features in OS Prediction}

In the univariate Cox analysis, the HPV status (positive: hazard ratio, 0.27; 95\% CI, 0.09-0.7; $P=.008$ ), T-stage ( $\geq$ III: hazard ratio, 3.66, 95\% CI, 1.34-9.99; $P=.01$ ), and rad-score (high-risk: hazard ratio, 3.72; 95\% CI, 1.21-11.46; $P=.02$ ) were found to be strongly associated with OS (Table 4). The Kaplan-Meier survival curves with logrank tests of individual variables are provided in the On-line Figure. The nomogram for 2- and 5-year OS probabilities is illustrated in Fig 4. For the multivariate Cox analysis, Cox model 1 ( $\mathrm{T}$ - and N-stage) had a concordance index of 0.621 ; the sequential addition of the HPV status and radscore to Cox model 1 increased the concordance index to 0.702 (Cox model 2) and 0.733 (Cox model 3), respectively. The internal and external validation of Cox model 3 yielded concordance indices of 0.866 and 0.720 , respectively. In addition,

Points

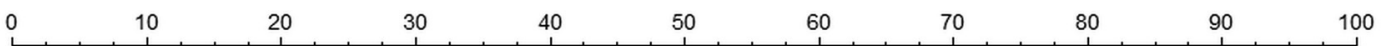

HPV_status

$$
\text { positive }
$$

rad_score

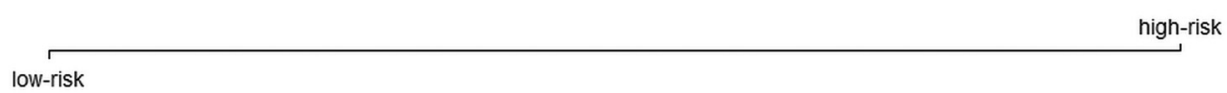

Total Points

\begin{tabular}{llllll}
\hline 50 & 100 & 150 & 200 & 250 & 300
\end{tabular}

2-year Sur. Prob.

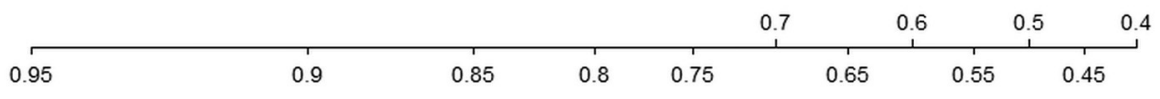

5-year Sur. Prob.

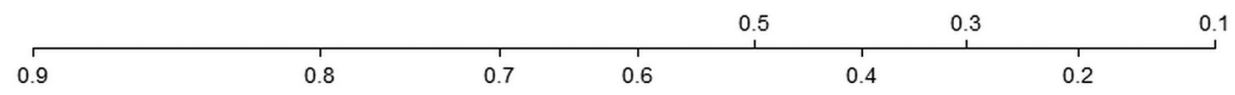

FIG 4. A nomogram of the 2- and 5-year overall survival. Sur. Prob. indicates survival probability. 


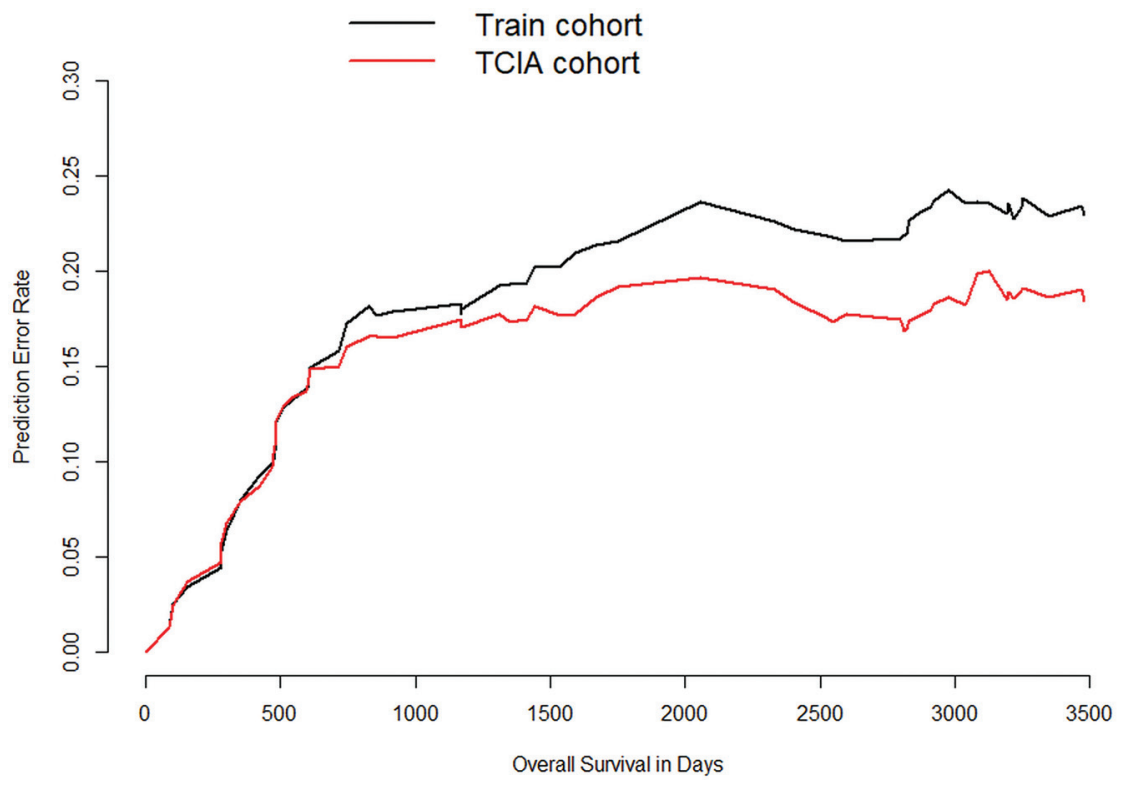

FIG 5. Prediction error curves with respect to the overall survival of the training (black line) and TCIA cohorts (red line).

Table 5: The integrated Brier scores of Cox model 3 in 2 cohorts

\begin{tabular}{lccl}
\hline Survival Time (Day) & Patients at Risk & Training Cohort & TCIA \\
\hline 0 & 78 & 0 & 0 \\
743 & 59 & 0.172 & 0.160 \\
2545 & 40 & 0.217 & 0.173 \\
3247 & 19 & 0.233 & 0.190 \\
\hline
\end{tabular}

the external validation of Cox model 3 onto the TCIA cohort resulted in a reduction in the prediction error rates (ie, TCIA cohort curve remaining below the training cohort curve [Fig 5]). Similarly, the integrated Brier score was lower in the TCIA cohort than in the training cohort for all OS periods (Table 5).

\section{DISCUSSION}

In the current study, quantitative pretreatment CT-based radiomics features were extracted from patients with primary OPSCC, and the subsequent analysis of these features yielded robust performance in the prediction of HPV status and OS. The results were reproduced reliably in both internal and external validation sets. The findings of this study provide preliminary evidence that pretreatment CT-based radiomics could potentially aid both HPV-status prediction and prognostication for patients with OPSCC.

Several studies have considered the implications of radiomics in head and neck cancers. The present data are consistent with prior similar studies in that radiomics demonstrated additive prognostic $^{13,14}$ and predictive value for HPV status. ${ }^{13,21}$ The studies performed by $\mathrm{Ou}$ et $\mathrm{al}^{13}$ and Bogowicz et $\mathrm{al}^{14}$ revealed that CT-based radiomics provided prognostic value in locally advanced head and neck cancers. However, the current study differs from theirs in that our target population was composed of untreated patients with OPSCC of various stages, which allowed more comprehensive risk assessment. Furthermore, our focused analysis on OPSCC minimized the potential heterogeneity that might be associated with various subtypes of head and neck cancers.

In multivariate Cox proportional hazards analysis, the addition of HPV status to the cancer stage increased the prognostic performance of the survival model. Likewise, the addition of the rad-score to the survival model fitted with HPV status and cancer stage further increased the prognostic performance. This finding suggests that radscore-when combined with HPV status and cancer stage-may provide an incremental benefit for prognostication of patients.

An interesting additional finding in this study was the good performance observed in the external validation set, which was better than that of the internal validation set. Compared with the institutional cohort, the external validation set represented a regionally different cohort with varying clinical variables. Regardless, the CT-based radiomics models trained with the institutional cohort exhibited comparable AUCs in HPVstatus prediction and better OS prediction in the external validation set. Therefore, a possible interpretation of this finding is that CTbased radiomics could be reproduced in multi-institutional cohorts.

As for the radiomics features, the most significant feature in OS prediction was the shape feature (spherical disproportion). This finding is consistent with a previous study by Leijenaar et $\mathrm{al},{ }^{12}$ who also found a shape feature as the most significant prognostic marker among other features associated with survival of patients with OPSCC. Additionally, a previous study found that primary head and neck cancers displaying asphericity on pretherapeutic $\left[{ }^{18} \mathrm{~F}\right]$ fluorodeoxyglucose uptake appeared to be associated with a poorer prognosis. ${ }^{22}$ Taken together, these findings suggest that the spatial heterogeneity of tumors might be associated with patients' prognosis. Future research should investigate the radiologic-pathologic correlation with regard to the spatial heterogeneity of the tumor.

The strength of the current study lies in its robust validations using internal and external datasets. While most previous radiomics studies of head and neck cancers used only internal validations $^{14}$ or lacked validations, ${ }^{23,24}$ we used both internal and external validations to minimize the potential overfitting of radiomics models. In addition, the primary OPSCC images were segmented in 3D rather than in circular ROIs on single axial slices. These volumetric segmentations encompassed the entire oropharyngeal squamous cell carcinoma (OPSCC) and, therefore, better reflected the true intratumoral environment.

There are several limitations to this study. First, selection bias might have been present due to the retrospective nature of the study. However, we attempted to minimize the potential selection bias by strictly adhering to the predefined selection criteria. 
Second, the issue of reproducibility is ongoing in most radiomics studies. ${ }^{25}$ Thus, the open-platform PyRadiomics was adopted as our primary source of radiomics features. Furthermore, the external validation set used in this study was acquired from the TCIA, the public repository of oncologic medical images, thereby increasing the chance of reproducibility of our results. Some of the variables (ie, OS, treatment received, HPV status) differed between our cohort and the TCIA cohort; however, the differences would not have affected the outcome because the Cox models were built from the training cohort and the TCIA was used solely for external validation.

\section{CONCLUSIONS}

The current study highlighted the feasibility of CT-based radiomics in HPV status and OS prediction for patients with OPSCC. The results were good and reliably validated in both internal and external datasets. Our findings add preliminary evidence to the potential value of CT-based radiomics in risk-stratification and treatment allocation for patients with OPSCC.

\section{ACKNOWLEDGMENT}

The authors appreciate the dataset generously provided by L. Wee and A. Dekker (https://doi.org/10.7937/tcia.2019.8kap372n).

\section{REFERENCES}

1. Pytynia KB, Dahlstrom KR, Sturgis EM. Epidemiology of HPV-associated oropharyngeal cancer. Oral Oncol 2014;50:380-86 CrossRef Medline

2. Ang KK, Harris J, Wheeler R, et al. Human papillomavirus and survival of patients with oropharyngeal cancer. $N$ Engl J Med 2010;363:24-35 CrossRef Medline

3. Chaturvedi AK, Engels EA, Pfeiffer RM, et al. Human papillomavirus and rising oropharyngeal cancer incidence in the United States. JCO 2011;29:4294-4301 CrossRef Medline

4. Ragin CC, Taioli E. Survival of squamous cell carcinoma of the head and neck in relation to human papillomavirus infection: review and meta-analysis. Int J Cancer 2007;121:1813-20 CrossRef Medline

5. O'Sullivan B, Huang SH, Siu LL, et al. Deintensification candidate subgroups in human papillomavirus-related oropharyngeal cancer according to minimal risk of distant metastasis. J Clin Oncol 2013;31:543-50 CrossRef Medline

6. Huang $\mathrm{SH}, \mathrm{Xu} \mathrm{W}$, Waldron J, et al. Refining American Joint Committee on Cancer/Union for International Cancer Control TNM stage and prognostic groups for human papillomavirusrelated oropharyngeal carcinomas. J Clin Oncol 2015;33:836-45 CrossRef Medline

7. O'Sullivan B, Huang SH, Su J, et al. Development and validation of a staging system for HPV-related oropharyngeal cancer by the International Collaboration on Oropharyngeal Cancer Network for Staging (ICON-S): a multicentre cohort study. Lancet Oncol 2016;17:440-51 CrossRef Medline

8. Gillies RJ, Kinahan PE, Hricak H. Radiomics: images are more than pictures: they are data. Radiology 2016;278:563-77 CrossRef Medline

9. Ravanelli M, Grammatica A, Tononcelli E, et al. Correlation between human papillomavirus status and quantitative MR imaging parameters including diffusion-weighted imaging and texture features in oropharyngeal carcinoma. AJNR Am J Neuroradiol 2018;39:1878-83 CrossRef Medline

10. Driessen JP, Caldas-Magalhaes J, Janssen LM, et al. Diffusionweighted MR imaging in laryngeal and hypopharyngeal carcinoma: association between apparent diffusion coefficient and histologic findings. Radiology 2014;272:456-63 CrossRef Medline

11. Westra WH. The morphologic profile of HPV-related head and neck squamous carcinoma: implications for diagnosis, prognosis, and clinical management. Head Neck Pathol 2012;6(Supple 1):48-54 CrossRef Medline

12. Leijenaar RT, Carvalho S, Hoebers FJ, et al. External validation of a prognostic CT-based radiomic signature in oropharyngeal squamous cell carcinoma. Acta Oncol 2015;54:1423-29 CrossRef Medline

13. Ou D, Blanchard P, Rosellini S, et al. Predictive and prognostic value of CT based radiomics signature in locally advanced head and neck cancers patients treated with concurrent chemoradiotherapy or bioradiotherapy and its added value to human papillomavirus status. Oral Oncol 2017;71:150-55 CrossRef Medline

14. Bogowicz M, Riesterer O, Ikenberg K, et al. Computed tomography radiomics predicts HPV status and local tumor control after definitive radiochemotherapy in head and neck squamous cell carcinoma. Int J Radiat Oncol Biol Phys 2017;99:921-98 CrossRef Medline

15. Ren J, Tian J, Yuan Y, et al. Magnetic resonance imaging based radiomics signature for the preoperative discrimination of stage III and III-IV head and neck squamous cell carcinoma. Eur J Radiol 2018;106:1-6 CrossRef Medline

16. Aerts HJ, Velazquez ER, Leijenaar RT, et al. Decoding tumour phenotype by noninvasive imaging using a quantitative radiomics approach. Nat Commun 2014;5:4006 CrossRef Medline

17. Moltz JH, Bornemann L, Kuhnigk J-M, et al. Advanced segmentation techniques for lung nodules, liver metastases, and enlarged lymph nodes in CT scans. IEEE J Sel Top Signal Process 2009;3:12234 CrossRef

18. van Griethuysen JJ, Fedorov A, Parmar C, et al. Computational radiomics system to decode the radiographic phenotype. Cancer Res 2017;77:e104-07 CrossRef Medline

19. Kursa MB, Rudnicki WR. Feature selection with the Boruta package. J Stat Softw 2010;36:1-13 CrossRef

20. Sauerbrei W, Royston $P$, Binder H. Selection of important variables and determination of functional form for continuous predictors in multivariable model building. Statist Med 2007;26:5512-28 CrossRef Medline

21. Huang C, Cintra M, Brennan K, et al. Development and validation of radiomic signatures of head and neck squamous cell carcinoma molecular features and subtypes. EBioMedicine 2019;45:70-80 CrossRef Medline

22. Apostolova I, Steffen IG, Wedel F, et al. Asphericity of pretherapeutic tumour FDG uptake provides independent prognostic value in head-and-neck cancer. Eur Radiol 2014;24:2077-87 CrossRef Medline

23. Buch K, Fujita A, Li B, et al. Using texture analysis to determine human papillomavirus status of oropharyngeal squamous cell carcinomas on CT. AJNR Am J Neuroradiol 2015;36:1343-48 CrossRef Medline

24. Fujita A, Buch K, Li B, et al. Difference between HPV-positive and HPV-negative non-oropharyngeal head and neck cancer: texture analysis features on CT. J Compu Assist Tomogr 2016;40:43-47 CrossRef Medline

25. Traverso A, Wee L, Dekker A, et al. Repeatability and reproducibility of radiomic features: a systematic review. Int J Radiat Oncol Biol Phys 2018;102:1143-58 CrossRef Medline 